\title{
Microstructural effects in the simulation of creep of concrete
}

\author{
Alain B. Giorla ${ }^{\mathrm{a}, *}$, Cyrille F. Dunant ${ }^{1}$ \\ ${ }^{a}$ Oak Ridge National Laboratory, One Bethel Valley Road, Oak Ridge, TN 37831-6148, USA \\ ${ }^{b}$ Department of Engineering, University of Cambridge, Trumpington Street, Cambridge CB2 1PZ, UK
}

\begin{abstract}
The influence of the microstructure on the visco-elastic properties of concrete is investigated using finite element simulations at the meso-scale. We first derive a constitutive law for the creep of the cement paste which accounts for both recoverable and permanent deformations, as well as the influence of temperature and internal relative humidity. The model is calibrated on a set of experiments at the cement paste scale, and then validated after upscaling to the concrete scale. The model is then applied to study the influence of the microstructure on the macroscopic creep of concrete. We show that materials with finer particles exhibit less creep, and that the anisotropy of creep can be explained with the shape and orientation of the aggregates. Furthermore, the acceleration of the stress relaxation in the presence of damage is explained by the micro-mechanical interaction between the aggregates, the cement paste, and the micro-cracks.
\end{abstract}

Keywords: Concrete; Creep; Finite Element; Microstructures;

\section{Introduction}

Concrete is the most used material in the world, and therefore a good understanding of its long-term behaviour is required for sustainability. Long-term behaviour of concrete depends both on durability aspects, e.g. alkali-silica reaction (ASR), freeze-thaw cycles, carbonation, and on its viscous properties. Both these aspects are critical in structures such as nuclear or hydraulic power plants which have long planned lives and are further subject to particular safety concerns. Models predictive over the long term require understanding the phenomena affecting the structures of interest at a fundamental level. In particular, behaviours should be derived from the complex processes that occur at the microstructure level.

Increasingly, concrete degradation phenomena are approached with numerical methods in which concrete is modelled as a twoor three-phase composite containing aggregates, cement paste and perhaps a transition zone between them. Examples include prediction of the material strength [1-9], visco-elastic properties [10-12], drying shrinkage [13, 14], or damage due to ASR [1517], high temperature [18], leaching [19], or irradiation [20, 21]. These multi-scale models are based on our best understanding of the physical processes concrete undergoes, therefore, they are suitable for long-term extrapolation as well as interpretation of experimental results.

The accuracy and predictive ability of such meso-scale models is strongly tied to the choice of constitutive behaviour which represent the various physical processes in the material. A primary purpose of these models is to provide insights on the phenomena occurring at the micro-scale, as well as characterizing

\footnotetext{
*Corresponding author:

Email address: a lain.b.giorla@gmail.com (Alain B. Giorla)
}

the interactions between the aggregates and the cement paste. Therefore, the absence of certain aspects in the constitutive behaviour might have a strong influence on the simulated results and on the conclusions one might draw from them. Notably, most models at this scale neglect the visco-elasticity of the cement paste, or model it as fully decoupled, considering that the driving mechanisms of interest have a much higher (or slower) rates than creep. However, this is not necessarily the case as the influence of creep can be seen from very short to very long time scales. At short time scale, the visco-elastic nature of the material leads to a strong dependency between the failure behaviour and the loading rate [3, 22, 23], and is also known to mitigate early-age shrinkage cracking risks [24-26]. At very long term, the recent study of the authors on the role of creep in ASR and irradiation showed that the stress relaxation of the cement paste also delays in the long run the propagation of the damage in the microstructure [16, 27].

While several models for creep exist in the literature, either at the macro-scale [28, 32, 34] or at the scale of the calciumsilicate hydrate $(\mathrm{C}-\mathrm{S}-\mathrm{H})$ phase in which the creep phenomenon occurs [35- 39], the underlying physical mechanism remains poorly understood and subject to debate and on-going research [28, 40, 42, 29, 30]. The separation into two main processes is commonly accepted:

- a reversible process involving the sliding of C-S-H structures one on another, in some models this is complemented with water movements in the gel pores,

- an irreversible process caused by changes in the $\mathrm{C}-\mathrm{S}-\mathrm{H}$ micro-structural arrangement.

The first mechanism controls the short-term creep, and is typically represented at the $\mathrm{C}-\mathrm{S}-\mathrm{H}$ scale with a linear dash-pot. 
Table 1: Slipping-compaction mechanisms in different creep theories.

\begin{tabular}{l|l|l}
\hline Theory & Decohesion & Hardening \\
\hline $\begin{array}{l}\text { Micro-prestress } \\
{[28]}\end{array}$ & breaking of high-stress atomic bonds & $\begin{array}{l}\text { redistribution of the stress over the microstruc- } \\
\text { ture }\end{array}$ \\
\hline $\begin{array}{l}\text { Nano-granular } \\
{[29]}\end{array}$ & displacement of C-S-H grains into empty spaces & self-compacting of the microstructure \\
\hline $\begin{array}{l}\text { Micro-crack } \\
{[30]}\end{array}$ & $\begin{array}{l}\text { displacement of water out of existing cracks and } \\
\text { pores }\end{array}$ & $\begin{array}{l}\text { displacement of water into the newly-formed } \\
\text { cracks }\end{array}$ \\
\hline $\begin{array}{l}\text { Dissolution-precipitation } \\
{[31]}\end{array}$ & $\begin{array}{l}\text { dissolution-precipitation of crystals under pres- } \\
\text { sure }\end{array}$ & increase in the affected interfaces \\
\hline
\end{tabular}

It is assumed to derive from the viscous sliding of C-S-H structures on each other. These may be the $\mathrm{C}-\mathrm{S}-\mathrm{H}$ sheets but also the $\mathrm{C}-\mathrm{S}-\mathrm{H}$ needles forming the bulk of the microstructure. In a previous work, Shahidi et al. [43] showed that the viscous behaviour of penny-shaped interfaces translates into an equivalent Kelvin-Voigt model at the upper scale using an analytic micro-mechanical homogenization scheme. The spring added in parallel at the cement paste level corresponds to the restraint caused by the elastic phases. The B3 model [28] and its various derivatives assume that this deformation spans a wide range of characteristic times following a progressive solidification theory. In a subsequent model, Hilaire et al. [34] used a single characteristic time, which seems more appropriate considering that Bazzoni [44] found that the C-S-H particles forms as needles of a single size characteristic of the binder, and Müller [45] showed that the gel intrinsic porosity also has a single characteristic size. Irfan-ul Hassan et al. [46] use an automated setup to identify the creep properties of cement paste during hydration. Their creep tests last 3 min., and they report no irreversibility in the creep.

The second mechanism controls the long-term creep and is assumed to be a logarithmic function of time. This behaviour has been identified at both the macro-scale [47] and the microscale with nano-indentation on mature C-S-H [29]. Different mechanisms have been proposed, which are all based upon modifications of the C-S-H microstructure.

In the micro-prestress theory proposed by Bažant et al. [28], it is assumed that the material is subject to very high localized stresses, which are progressively relaxed over time and redistributed throughout the microstructure even in absence of external loads. However, Gawin et al. [32] showed that this assumption is not sufficient to simulate the drying creep of concrete, and found better agreement by assuming that relative humidity plays a driving role in the creep deformation: they use a concept of effective stress used in poro-mechanics ( $\operatorname{sigma}_{e}^{s}=\sigma+\alpha p^{s} \mathbf{I}, \alpha$ Biot's coefficient, $\sigma$ total stress, and $p^{s}$ solid pressure) to calculate the creep deformation. Jirásek and Havlásek [33] considered a similar assumption by using a differential equation between the apparent viscosity of the material and the temperature and relative humidity variations.

In the nano-granular theory proposed by Vandamme and Ulm [29], derived from an analogy with the mechanical behaviour of soils, creep originates from the dislocation of $\mathrm{C}-\mathrm{S}-\mathrm{H}$ particles and their displacement into an adjacent empty pore of sufficient size. This causes a time-dependent deformation of the $\mathrm{C}-\mathrm{S}-\mathrm{H}$ micro-structure. As the deformation increases, the space available diminishes which leads to a progressive deceleration of the creep.

Rossi et al. [30] proposed a combination of micro-cracking and water diffusion into the newly-opened cracks to explain creep. The displacement of water from one crack to another reduces the cohesive force at the crack tip, allowing it to further propagate and leading to a load-induced drying shrinkage. This is consistent with the occurrence of acoustic emissions observed during a creep experiment as well as the non-linearity of creep for high level of load, but the authors did not provide a mathematical model to support their theory.

Finally, Pignatelli and colleagues [31] proposed a model in which C-S-H crystals, locally under load through the contact of other crystals dissolve and precipitate where there is no contact. Creep occurs as $\mathrm{C}-\mathrm{S}-\mathrm{H}$ needles interpenetrate through this mechanism. As this causes the microstructure to compact, the long-term creep differs from the short-term creep.

In all models, the long-term creep seems to be related to progressive slipping in the $\mathrm{C}-\mathrm{S}-\mathrm{H}$ microstructure, followed by its compaction. Table 1 identifies for each of the theory described above the mechanism corresponding to the slipping and compaction phases of the creep process. In the modelling, this can be represented as a time-dependent dashpot [28]. The separation of the behaviour in two processes is convenient from the point of view of modelling, but homogenisation of creeping composites suggests that since a single phase is responsible for the timedelayed behaviour, and does not exhibit measurable changes in its intrinsic behaviour over time, a single characteristic time should describe creep in the long and short term. The transition between apparent short-term and long-term behaviour is then linked to the irreversibility of the behaviour.

While the need to account both for long-term and shortterm behaviour is accepted now in the concrete community, few mesoscale models for concrete use it to describe the visco-elastic behaviour of the cement paste. In fact, the rare meso-scale models which actually account for creep at that scale approximate the cement paste behaviour with non-ageing Maxwell chains, either in the upscaling of visco-elasticity itself [10, 11] or in the analysis of discrete crack propagation in visco-elastic ma- 
terials [48, 3]. This linear representation of the material shows no irrecoverable deformation, and is therefore unable to capture some of the non-linear effects observed in concrete creep such as non-superposition or non-linearity at high levels of load (see for example [49]). These aspects are critical for long-term modelling of the material.

The constitutive equation for the visco-elastic behaviour of mature cement paste we propose relies on four parameters that can be easily read on a creep curve, provided it includes at least one unloading step. Importantly, the proposed model has a single characteristic time, but yields both long and short term behaviour compatible with the phenomenological observations reported in the literature. The behaviour proposed in this work is properly classified as an ageing linear visco-elastic material. Extensions are proposed to account for the influence of temperature and relative humidity. These effects are calibrated on independent experiments from the literature. Several examples of multi-scale analysis of concrete creep experiments are shown to validate the behaviour. The discussion emphasizes micro-structural effects on the apparent concrete behaviour, notably the influence of the shape and orientation of the inclusions. The model is integrated within the space-time finite element method presented by the authors in [50], as implemented in the $\mathrm{C}++$ finite element framework AMIE [15, 51]. We validate our model based on the experiments by Le Roy [52], and use it to interpret the results by Denarié and co-workers [3] who studied the coupling between damage and creep. A key finding of this paper is the identification of the role of the particle size distribution of the inclusions in creep properties.

\section{Constitutive behaviour of the cement paste}

We assume that creep originates from two separate and simultaneous mechanisms:

- a reversible sliding of the $\mathrm{C}-\mathrm{S}-\mathrm{H}$ structures one on another;

- an irreversible slipping and compaction of the C-S-H nanostructure.

The first mechanism controls the short-term creep, while the second is related to the long-term behaviour of the material. The strain $\epsilon$ is then written as the sum of the elastic strain $\epsilon_{e}$, the recoverable viscous strain caused by the sliding of the $\mathrm{C}-\mathrm{S}-\mathrm{H}$ $\boldsymbol{\epsilon}_{r}$, the irrecoverable viscous strain caused by the changes in the C-S-H nano-structure $\epsilon_{v}$, and additional deformations caused by changes in temperature $\epsilon_{t}$ or humidity $\epsilon_{h}$.

$$
\boldsymbol{\epsilon}=\boldsymbol{\epsilon}_{e}+\boldsymbol{\epsilon}_{r}+\boldsymbol{\epsilon}_{v}+\boldsymbol{\epsilon}_{t}+\boldsymbol{\epsilon}_{h}
$$

Elastic deformation. The relation between the elastic strain and the stress $\sigma$ is straightforward:

$$
\boldsymbol{\sigma}=\mathbb{C}_{e}: \boldsymbol{\epsilon}_{e}
$$

Where $\mathbb{C}_{e}$ is the fourth-order elastic tensor of the material, which can be derived either from experiments, analytic homogenization (see for example [53]) or numerical homogenization (see for example [39]).
Short-term creep. The recoverable creep is represented with a single Kelvin-Voigt unit in which the viscosity derives from the sliding of the C-S-H structures one on another, and the elastic spring accounts for the restraint provided by the elastic phases in the material:

$$
\boldsymbol{\sigma}=\mathbb{C}_{r}:\left[\boldsymbol{\epsilon}_{r}+\tau_{r} \dot{\boldsymbol{\epsilon}}_{r}\right]
$$

Where $\mathbb{C}_{r}$ is a fourth-order stiffness tensor which relates the stress to the final recoverable creep deformation. It can be read from the unloading section of a creep experiment, assuming the temperature and humidity are otherwise constants in the sample. Alternatively, it can be estimated using analytic homogenization models as in [43].

Long-term creep. The long-term creep originates from the slipping and compaction of the $\mathrm{C}-\mathrm{S}-\mathrm{H}$ nano-structure under load. The slipping mechanism induces a displacement rate proportional to the load, while the compaction mechanism impedes this deformation and increases the apparent viscosity of the material. The constitutive law is then:

$$
\boldsymbol{\sigma}=\gamma \mathbb{E}_{v}: \dot{\boldsymbol{\epsilon}}_{v}
$$

Where $\gamma$ represents the state of compaction of the $\mathrm{C}-\mathrm{S}-\mathrm{H}$ microstructure, and $\mathbb{E}_{v}$ the fourth-order viscosity tensor of the slipping mechanism before compaction.

This law can also be considered from a friction mechanics perspective: if $\gamma$ is a measure of the relative contact surface area of the microstructure, then $\sigma / \gamma$ gives a measure of the internal frictional force, and is proportional to the speed of the system $\dot{\boldsymbol{\epsilon}}_{v} \cdot \gamma$ does not measure the contact surface, rather it moves proportionally to it. We complement (4) with the evolution of the hardening variable in time. Assuming the analogy with the soils behaviour identified by Vandamme and colleagues [29, 38], we use the following rate evolution:

$$
\frac{\partial \gamma}{\partial t}=\frac{1}{\tau_{v}}
$$

Where $\tau_{v}$ is the characteristic time of the compaction process, and is a function of the temperature and relative humidity. (4 5 (5) introduce three $a$ priori independent parameters, but only two values are of practical interest: the initial viscosity $\gamma(t=0) \mathbb{E}_{v}$ and its evolution rate $\left(1 / \tau_{v}\right) \mathbb{E}_{v}$. We can freely set $\gamma(t=0)=1$. Inn this work $t=0$ corresponds to the time of loading.

Given that the recoverable and irrecoverable creep originate both from the same phase, we assume that these two processes have the same characteristic time:

$$
\tau_{r}=\tau_{v}
$$

Finally, the material behaviour reads, after combination of 
(146):

$$
\begin{gathered}
{\left[\begin{array}{ccc}
\mathbb{C}_{e} & -\mathbb{C}_{e} & -\mathbb{C}_{e} \\
-\mathbb{C}_{e} & \mathbb{C}_{e}+\mathbb{C}_{r} & \mathbb{C}_{e} \\
-\mathbb{C}_{e} & \mathbb{C}_{e} & \mathbb{C}_{e}
\end{array}\right]\left[\begin{array}{c}
\boldsymbol{\epsilon} \\
\boldsymbol{\epsilon}_{r} \\
\boldsymbol{\epsilon}_{v}
\end{array}\right]+} \\
{\left[\begin{array}{ccc}
0 & 0 & 0 \\
0 & \tau_{v} \mathbb{C}_{r} & 0 \\
0 & 0 & \gamma \\
\mathbb{E}_{v}
\end{array}\right]\left[\begin{array}{c}
\dot{\boldsymbol{\epsilon}} \\
\dot{\boldsymbol{\epsilon}}_{r} \\
\dot{\boldsymbol{\epsilon}}_{v}
\end{array}\right]=\left[\begin{array}{c}
\boldsymbol{\sigma}+\mathbb{C}_{e}:\left[\boldsymbol{\epsilon}_{t}+\boldsymbol{\epsilon}_{h}\right] \\
-\mathbb{C}_{e}:\left[\boldsymbol{\epsilon}_{t}+\boldsymbol{\epsilon}_{h}\right] \\
-\mathbb{C}_{e}:\left[\boldsymbol{\epsilon}_{t}+\boldsymbol{\epsilon}_{h}\right]
\end{array}\right]}
\end{gathered}
$$

Which can be easily integrated in the space-time finite element framework developed by the authors [50] or alternatively, with more common finite differences (first formulated in [54]). The space-time finite element method is equivalent to an implicit Euler scheme if the element faces are parallel to the time direction, or to any Newmark finite difference scheme with the right choice of trial function [55].

In (7), the forces induced by the thermal expansion and drying shrinkage also appear on the second (respectively third) line corresponding to the creep deformation. These terms originate from (3) (respectively (4)) to which the first line of (7) has been subtracted, yielding a symmetrical system.

Overall, these equations describe an ageing linear viscoelastic material.

\section{Identification of material parameters}

The material behaviour described above requires the knowledge of four different material properties:

- $\mathbb{C}_{e}$ which corresponds to the initial elastic deformation of the material,

- $\mathbb{C}_{r}$ which corresponds to the amplitude of the creep recovered during the unloading of the material,

- $\mathbb{E}_{v}$ which corresponds to the asymptotic slope of the creep curve in the logarithmic time,

- $\tau_{v}$ which marks the transition to logarithmic creep.

We assume that the material is isotropic, which leads to a decomposition of all fourth-order tensors using only two parameters: Young's modulus E and a Poisson ratio $\nu$ (or alternatively, a bulk and a shear modulus). However, only the Young's modulus of each tensor can be extracted from a standard uni-axial creep test. The apparent Poisson's ratio of the resulting visco-elastic material depends the choice of the rheological network, but also on the choice of definition of the Poisson's ratio [56, 57]. In this work, the Poisson's ratio of the visco-elastic composite should be understood as the asymptotic Poisson's ratio. After Aili and colleagues:

$$
\nu_{\infty}=\frac{3 K_{\infty}-2 G_{\infty}}{6 K_{\infty}+2 G_{\infty}}
$$

A typical creep curve is shown in Figure 1, including unloading curves at different instants of the deformation. From these curves the three values $\mathrm{E}_{e}, \mathrm{E}_{r}$ and $\mathrm{E}_{v}$ can immediately be identified from respectively the initial deformation, the recovered deformation, and the asymptotic logarithmic slope.

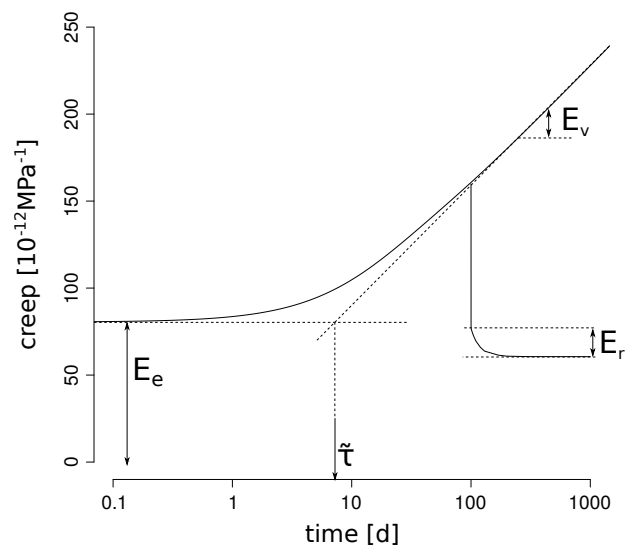

Figure 1: Example of creep curve with an unloading branch at 100 days, and identification of the four material parameters $\mathrm{E}_{e}, \mathrm{E}_{r}, \mathrm{E}_{v}$ and $\tilde{\tau}$.

However, the characteristic time $\tau_{v}$ does not appear immediately as the two creep process overlap in time. We can easily read the apparent characteristic time $\tilde{\tau}$ as the intersection between the initial horizontal asymptote (the elastic deformation) and the final logarithmic asymptote. As recoverable creep stops after a certain period of time, it is possible to obtain $\tau_{v}$ from $\tilde{\tau}$ and the ratio between the two viscosities:

$$
\tau_{v} \simeq \tilde{\tau} \exp \left(\frac{\mathrm{E}_{r}}{\mathrm{E}_{v}}\right)
$$

To interpret experiments from the literature, it is necessary to take into account temperature and relative humidity.

\section{Influence of temperature and relative humidity}

In this section, we estimate the influence of the temperature $T$ and relative humidity $h$ on the creep deformations. We assume that these two factors are not correlated: the temperature affects the rate of the creep deformation $\tau_{v}$, while the relative humidity affects its amplitude $\mathbb{C}_{r}$ and $\mathbb{E}_{v}$ :

$$
\begin{aligned}
\tau_{v} & =f(T) \tau_{v \mid T=T_{0}} \\
\mathbb{C}_{r} & =g(h) \mathbb{C}_{r \mid h=1} \\
\mathbb{E}_{v} & =g(h) \mathbb{E}_{v \mid h=1}
\end{aligned}
$$

Where $f$ and $g$ are two functions to identify. The temperature and relative humidity also affects the hydration of the cement, but these effects can be ignored in this model of mature concretes.

It is possible to account for drying creep after the method of Benboudjema and colleagues [26] by introducing an equivalent $\gamma$ :

$$
\gamma_{\mathrm{eq}}=\frac{1}{\frac{1}{\gamma}+\mu\left|\frac{\mathrm{d} h}{\mathrm{~d} t}\right|}
$$

This is equivalent to addng a second dashpot in series accounting for the effect of drying. 


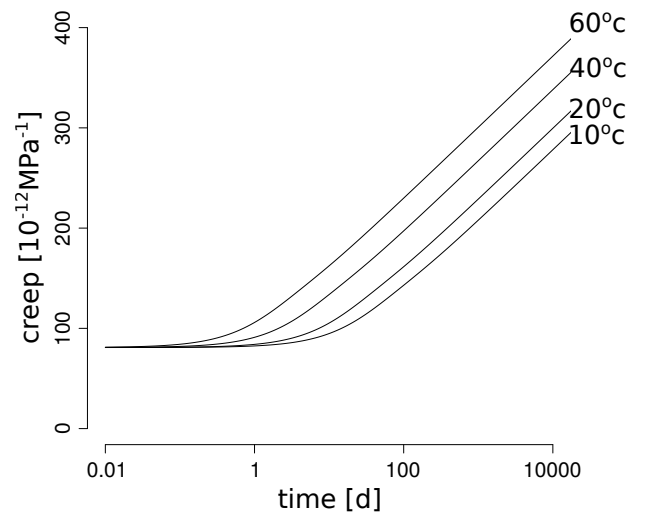

Figure 2: Effect of temperature on the creep deformation.

\subsection{Influence of temperature}

It is commonly admitted that creep is accelerated when increasing the temperature following an Arrhenius-like law (see for example [49, 58]).

$$
f(T)=\exp \left(T_{a}\left(\frac{1}{T}-\frac{1}{T_{0}}\right)\right)
$$

Where $T_{a}$ is the "activation temperature" for creep and $T_{0}$ the reference temperature. The value of $T_{a}$ seems to vary considerably from one cement to another, as Bažant et al. [58] suggest $5000 \mathrm{~K}$, but Bengougam [59] found values ranging from 6000 to $17000 \mathrm{~K}$ for cores extracted from concrete dams. In both cases, these values were measured for concrete and not cement paste. Examples of creep curves for cement paste at different temperatures (using $T_{a}=5000 \mathrm{~K}$ ) are shown in Figure 2

\subsection{Influence of relative humidity}

Creep of concrete is strongly related to the state of water in the $\mathrm{C}-\mathrm{S}-\mathrm{H}$, and several studies have aimed at characterising the creep as a function of the amount of water. Wittmann [60] studied the creep of sealed cement paste specimens pre-dried at a relative humidity ranging from 0 to 0.985 . We fitted each creep curve separately using the same characteristic time (found equal to 2 days) and $\mathrm{E}_{r}=\mathrm{E}_{v}$. The evolution of the $\mathrm{E}_{v} / \mathrm{E}_{v}(h=$ 0.985 ) against the relative humidity is shown in Figure 3 .

From this curve, we identified the following relation between the creep properties and the humidity:

$$
g(h)=\frac{1-h}{h_{0}}+\exp \left(\frac{h-1}{h_{0}}\right)
$$

Where $h_{0}$ is a coefficient that was fit and found equal to 0.2 in this specific set of experiments. However, it is likely that this coefficient depends from the material microstructure, and might change with different materials or age.

The main model parameters for typical cement paste are summarized on Table 2 .

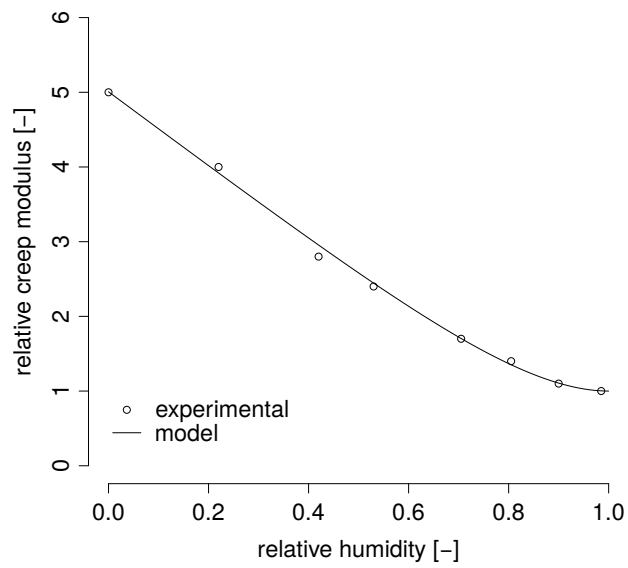

Figure 3: Effect of relative humidity on creep modulus of cement paste. Experimental data from [60].

\section{Model validation}

We use the experimental results of the extensive study of Le Roy [52] to validate the model developed above. Le Roy measured the basic creep of a large set of concretes, varying the cement paste composition, aggregate content, or water/cement $(w / c)$ ratio. He also measured the creep of the corresponding cement pastes.

We use the experimental creep curves for cement paste to calibrate our model, and then perform a numerical creep test on a concrete microstructure with the same cement properties, and compare the creep of the simulated concrete with the corresponding experimental value.

The composition of the three concretes is shown in Table 3 . where $w / c$ is the water-to-cement ratio, SF the amount of silica fume added to the mix (as a mass fraction of cement), and $f_{g}$ the aggregate volumetric fraction. The samples were loaded at 28 days and sealed, so we ignore hydration effects as well as shrinkage in this study.

Cement paste. The material properties for the cement paste are obtained from the basic creep curves measured for the corresponding paste. We assume the scalar playing the role of a Poisson ratio of all tensors in the material model $\nu_{e}, \nu_{r}, \nu_{v}$ to be equal to 0.2 as an arbitrary value since only uni-axial creep curves produced in this work. Furthermore, we also assume $\mathrm{E}_{r}=\mathrm{E}_{v}$ in absence of appropriate unloading data. This leaves only three parameters which are easily read on the experimental curves.

Aggregates. The aggregates are simulated as purely elastic materials with a Young's modulus of $77 \mathrm{GPa}$ as measured by Le Roy, and a Poisson ratio of 0.2 in absence of further data.

Sample and microstructure generation. The samples used in the concrete creep experiments were cylinders $16 \mathrm{~cm}$ in diameter and $100 \mathrm{~cm}$ in length. Since we cannot simulate both the entire sample and the entire aggregate particle size distribution, we limit ourselves to a $8 \times 8 \mathrm{~cm}$ square slices of the same sample, 
Table 2: Typical model parameters for cement paste.

\begin{tabular}{crll}
\hline Parameter & Value & Unit & Reference \\
\hline $\mathrm{E}_{e}$ & 12 & $\mathrm{GPa}$ & Calibrated on creep experiments by [52] \\
$\nu_{e}$ & 0.2 & {$[-]$} & Typical value for cement and concrete \\
$\mathrm{E}_{v}$ & 30 & $\mathrm{GPa} \cdot \mathrm{d}$ & Calibrated on creep experiments by [52] \\
$\tau_{v}$ & 3.3 & $\mathrm{~d}$ & Calibrated on creep experiments by [52] \\
$T_{a}$ & 5000 & $\mathrm{~K}$ & Typical value for concrete recommended by [58] \\
$h_{0}$ & 0.2 & {$[-]$} & Calibrated on creep experiments by [60] \\
\hline
\end{tabular}

Table 3: Composition of the concrete mixes and material parameters for the corresponding cement pastes from [52].

\begin{tabular}{r|ccc|ccc}
\hline Mix & w/c & SF & $f_{g}$ & $\mathrm{E}_{e}[\mathrm{GPa}]$ & $\mathrm{E}_{v}[\mathrm{GPa} \cdot \mathrm{d}]$ & $\tau_{v}[\mathrm{~d}]$ \\
\hline B0 & 0.5 & 0 & 0.705 & 12 & 30 & 3.3 \\
B6 & 0.28 & 0.1 & 0.71 & 25 & 70 & 4.1 \\
B7 & 0.38 & 0.1 & 0.713 & 17 & 80 & 2.5 \\
\hline
\end{tabular}

in which the entire particle size distribution of the material is represented. The left edge of the simulated sample corresponds to the vertical axis of the cylinder, so its horizontal displacement is set to 0 . The aggregate particle size distribution in the simulation follows the distribution measured given by Le Roy, and the volume fraction of aggregates is the same in the simulations and in the experiments. The boundary conditions and simulated microstructure for the B0 concrete are shown in Figure 4. The samples are two-dimensional, and the plane strain approximation is used.

Results. Figure 4 shows on the top the experimental creep curve for the cement paste and the model calibrated using the values found in Table 3 , and the experimental and numerical creep curves at the concrete level on the bottom. We obtain a good agreement between the experimental and simulated creep, except for the concrete B6 for which the model predict a higher creep. However, this could be related to additional shrinkage, since this specific material has a very low water/cement ratio. Indeed, Le Roy noticed a very high autogeneous shrinkage in this material. This may further explain why the identified parameters do not follow a monotonic trend. Königsberger and colleagues proposed a model which would give the parameters we use based on the cement composition [61]. Previous work by this same author suggest a method to further predict strength [62, 63]. Such an approach is an important step towards predicting all concrete properties from its formulation. In this instance, the approach would fail as some factor caused one of the mixes to misbehave. Even then, the parameters of the proposed model can be identified from the creep experiment.

\section{Microstructure effects in concrete creep}

We use the model to estimate the influence of the concrete microstructure on the macroscopic creep. In all cases, we use the cement paste and aggregate mechanical properties calibrated on Le Roy's B0 experiment.

\subsection{Particle size distribution}

The particle size distribution plays a vital role in some degradation processes in concrete, as shown for example in the case of fracture [6], ASR [64], or drying shrinkage [14]. The question is also raised for creep, and cannot generally be assessed by analytic homogenization scheme without additional refinement like the tri-sphere model proposed by Le Roy [52]. In a recent paper, Lavergne et al. computed apparent properties of composites simulated in $3 \mathrm{D}[12]$. They found that the macroscopic creep properties were somehow independent of the particle size distribution, but in their work, the ratio between the smallest and largest particle was around 1/10, while the particle size distribution of aggregates in concrete spans two orders of magnitude.

Zhang and colleagues noted that the characteristic time of creep for pastes and concretes differ [65]. Indeed, we observe that the inclusions increase the apparent characteristic time of the material. The definition of characteristic time used in the latter work is not the same as in this one, nonetheless, the observation stands. Here, we test four different particle size distribution: a continuous particle size distribution using the standard Bolomey [66] curve used in Swiss construction, and three discontinuous distributions containing a majority of large, medium or small aggregates. In the case of the distribution with a majority of small aggregates, an additional class of smaller aggregates was added to make possible the random generation of the microstructure. The volume fraction of the cement paste is kept the same in all simulations. The mesh was generated with a density fine enough to capture even the smallest inclusions.

In principle, there are no clear separations of scale in concrete, as the largest cement grains are close in size to the smallest sand grains. However, a clearer separation between the aggregates and paste emerges at later ages, as the cement grains become fully hydrated and the outer $\mathrm{C}-\mathrm{S}-\mathrm{H}$ becomes nearly as dense as the inner. As the model proposed here is more readily applicable to mature paste, we believe it is a complete representation of a $2 \mathrm{D}$ slice of the microstructure. 


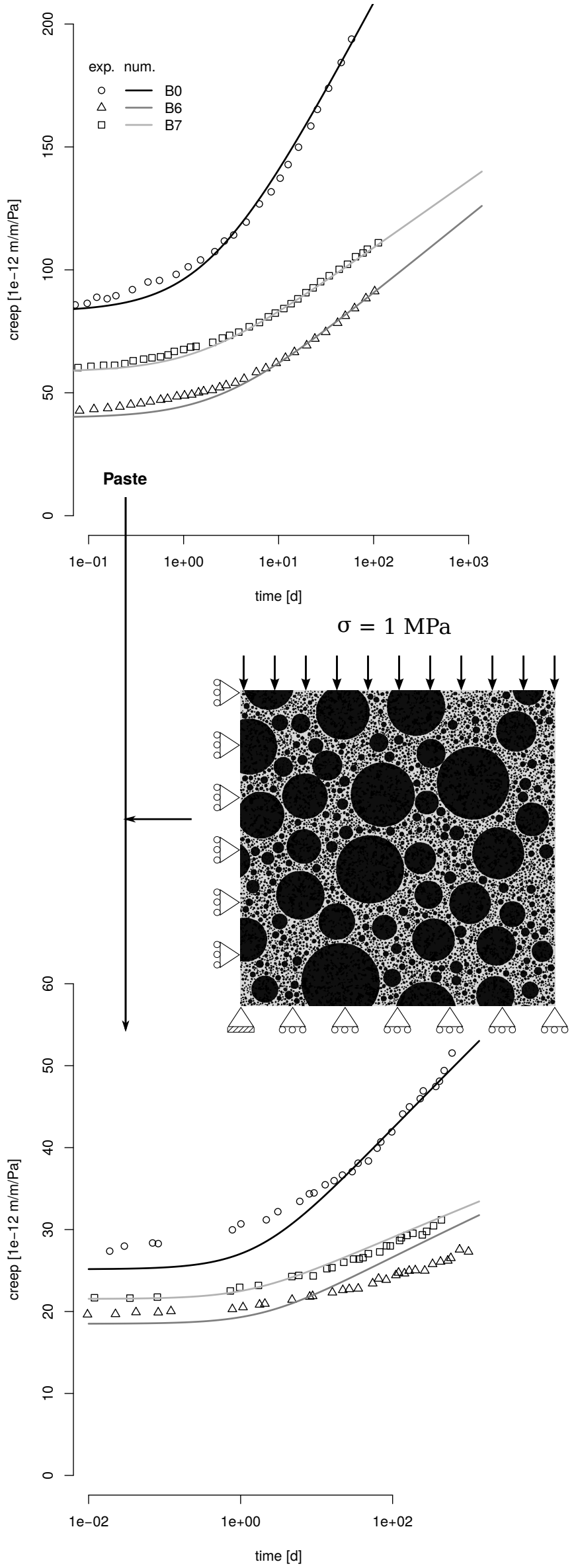

Figure 4: Experimental and numerical creep curves for cement paste (top) and 7 concrete (bottom). Experimental data from [52]. The microstructure for the concrete $\mathrm{B} 0$ and the boundary conditions applied to the numerical concrete sample are shown in the middle.
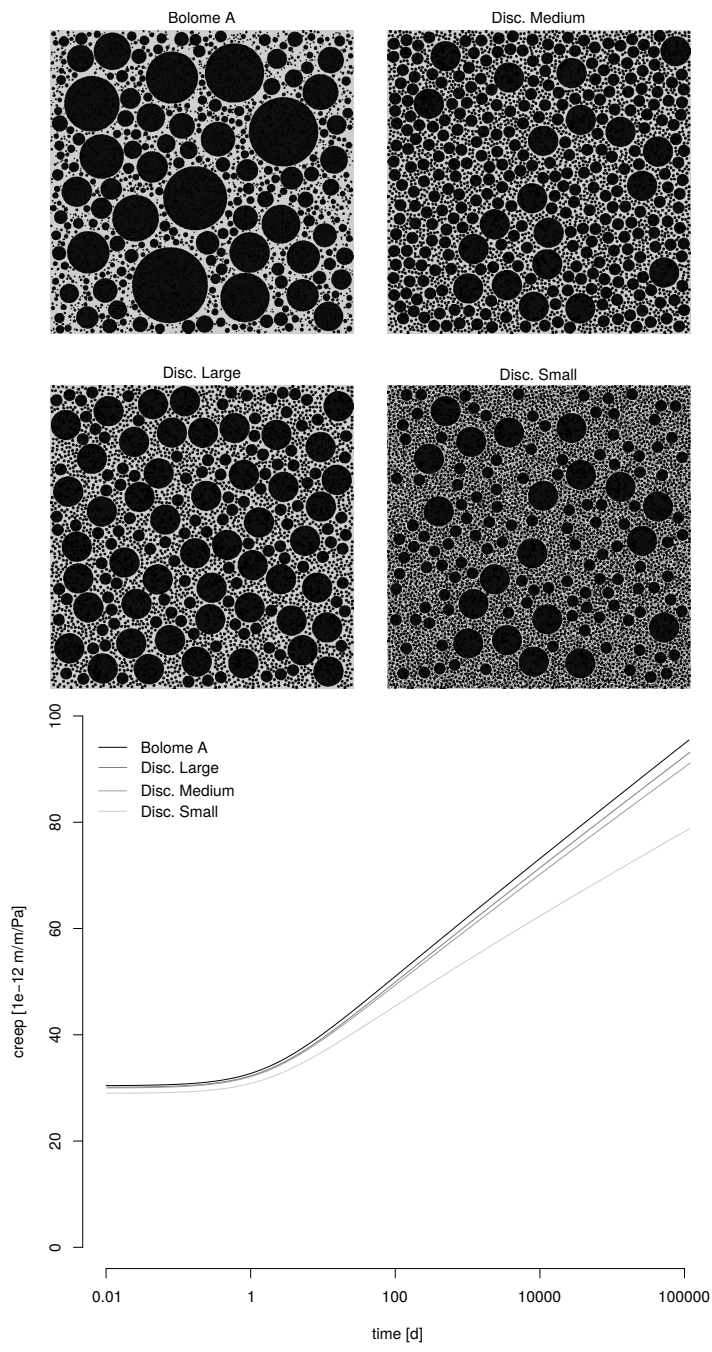

Figure 5: Numerical simulations of creep with four different particle size distribution.

The uni-axial creep compliances for the four distributions are shown in Figure 5 alongside their respective microstructures. The microstructures containing the larger aggregates show the higher creep, while a large amount of fine particles seem to be able to reduce the creep. This effect is particularly significant with the smallest particle size distribution, and shows the role of the smallest particles in controlling the creep deformation. All four simulations were done with a mesh fine enough to represent even the smallest aggregates, and the relative difference in area between the theoretical aggregate distribution and the distribution after meshing is lower than $0.1 \%$ in all cases.

This is of practical relevance for numerical models of concrete at the meso-scale, since it indicates that the creep or stress relaxation of the material might be overestimated if the smallest aggregates are not accounted for in the simulation. A similar effect have been shown for fracture mechanics [6], and therefore meso-scale simulations with creep and damage should reproduce the entire particle size distribution of the aggregates as close as possible. 


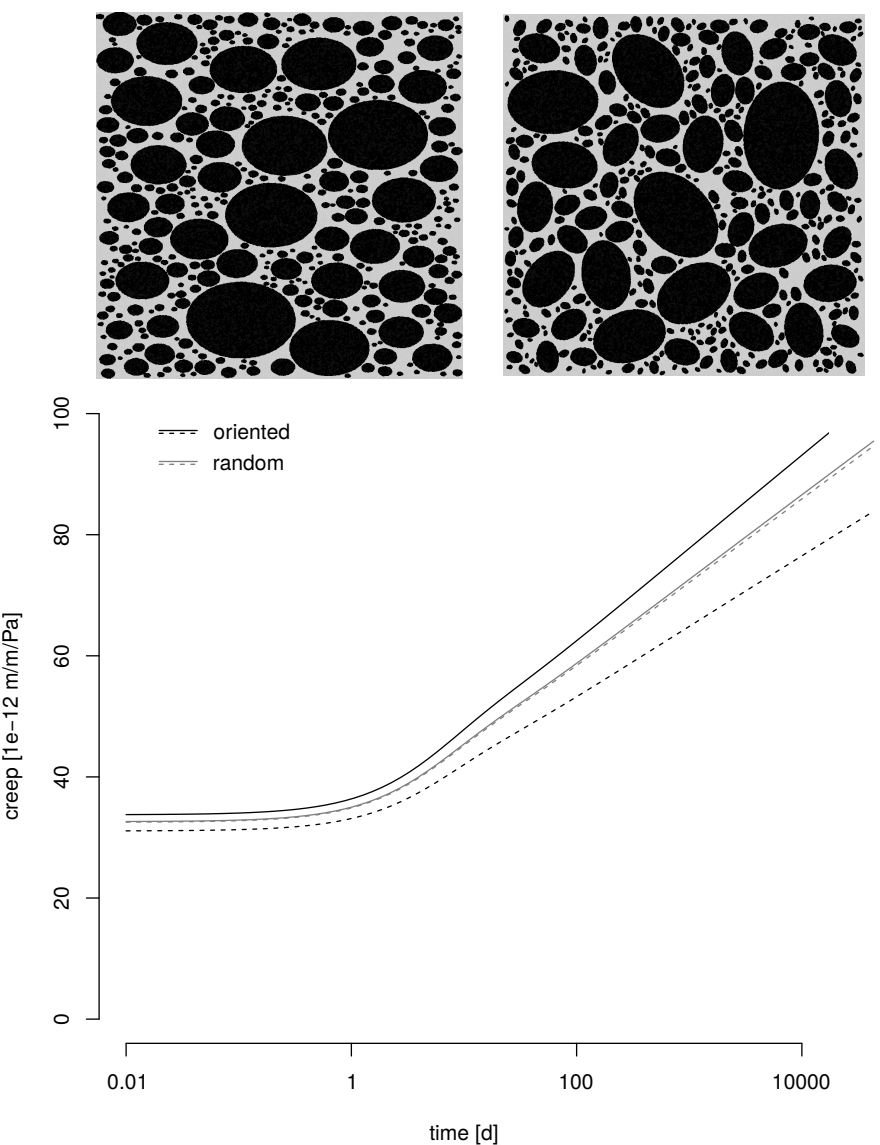

Figure 6: Numerical simulations of creep with aligned or randomly oriented ellipses, loaded along the vertical axis (solid lines) or the horizontal axis (dashed lines).

\subsection{Particle shape and orientation}

Concrete is typically assumed isotropic, even though a certain anisotropy can be measured with respect to the casting direction. In the case of creep, the lateral creep is somewhat inferior to the axial creep [49]. In meso-scale simulations, aggregates are typically simulated as circles or spheres, as they are the easiest shape to manipulate, which however cannot provide an anisotropic behaviour. Notable exceptions include works with randomly generated polygonal aggregates [2, 4, 13], or using images of the material microstructure [1, 67, 12].

In the present work, we use ellipses to represent the casting direction of the aggregates. The ellipses are generated with an aspect ratio (minor radius / major radius) equal to 0.7 . We simulate two microstructures with the same particle size distribution, one in which the ellipses are randomly oriented, and a second one in which the principal axis of the ellipse is preferably oriented along the horizontal axis. On each microstructure, we perform two different creep experiments, one in the axial direction, and one in the lateral direction.

The resulting creep curves as well as the microstructures are shown in Figure 6. As expected, the microstructure with the randomly oriented ellipses gives an isotropic response, while the microstructure with the oriented ellipses leads to an anisotropic creep with a similar extent to what is reported in [49]. However, the lack of comprehensive experimental data on aggregate shape, cement paste creep and corresponding concrete creep makes this result difficult to properly evaluate.

The effect of particle shape and orientation becomes prominent when multi-axial loading are considered. Indeed, several studies have shown that the bulk and shear creep do not occur at the same rate nor with the same magnitude, both at the concrete level and at the cement paste level.

For the paste, several studies have shown that the bulk and shear creep deformations occur at different rates and magnitudes. Unfortunately, the experimental results of Bernard and co-workers [68] were obtained on leached cement paste, which makes questionable the application to sound cement paste, while the experiments of Grasley et al. [69] were at very early age, where hydration effects dominate the mechanical and shrinkage behaviour of the material. Therefore, we did not attempt to simulate these experiments.

At the concrete level, the difference in volumetric and deviatoric creep interacts with the effect of the shape and orientation of the particles. This last information is generally missing in the multi-axial creep experiments from the literature, which makes at this stage difficult the uncoupling between the two phenomena. For these reasons, no attempt were made to simulate this phenomena with the present model.

\section{Effect of damage on creep}

Bažant and Gettu [22] found that the apparent relaxation of concrete is accelerated when damage in the material increases. They proposed to explain this effect with the increased size of the fracture process zone as a function of the pre-peak load. However, Denarié and colleagues [3] found in a later study a similar trend on samples which where all loaded at the same rate, and therefore in which the fracture process zone had the same characteristic length. Therefore, another effect seems necessary to understand this phenomena.

We simulate the relaxation of concrete specimens in which damage was artificially introduced. We use the same microstructure and material properties as for the simulations of Le Roy [52] experiments. These are not coupled creep-damage simulations, as we assume that the relaxation setup does not increase the damage in the material. The damage is calculated beforehand with a simplified approach. The goal of these simulations is to estimate micro-structural effects on the apparent relaxation properties, and not characterize the effect of creep on the progress of damage. Important work by Nguyen and colleagues [70] show that the effect of damage on the creep components, assuming randomly oriented microcracks, is the same as what would be predicted by a Mori-Tanaka scheme. This indicates that the treatment of damage as proposed in this paper - in the paste only is representative of the results which would be obtained using a multi-level homogenisation approach.

Five different microstructures are generated using the following procedure:

1. An elastic tension test is performed on the numerical sample. 
2. The strains in the cement paste are averaged over a certain process zone to account for non-local damage effects [71].

3. The elements located in the cement paste with an averaged strain higher than $99 \%$ of the highest strain in the sample are removed from the finite element assembly.

4. Steps 1-3 are repeated until a certain fraction of damage in the microstructure is reached.

The $1 \%$ tolerance chosen at step 3 . of the procedure ensures the formation of micro-cracks homogeneously distributed through the paste, as opposed to a single macro-crack crossing the sample. In addition, the elements located at the top of the sample are prevented from cracking in order to be able to apply homogeneously the boundary conditions. Five other microstructures are generated by randomly placing circular pores in a homogeneous material. The mesh density in the homogeneous material is the same as for the concrete microstructure.

After convergence of the damage computation, the vertical displacement on the upper boundary of the sample is maintained constant to measure the relaxation properties of the damaged material. From this test we measure the normalized relaxation of that specimen as the loss of stress over time:

$$
\mathcal{R}(d, t)=\frac{\sigma_{22}(d, t)}{\sigma_{22}(d, 0)}
$$

To observe the influence of damage over the relaxation behaviour, we plot $\mathcal{R}(d, t) / \mathcal{R}(0, t)$ for both scenarios (concretelike microstructure and homogeneous microstructure) in Figure 7, alongside experimental relaxation curves measured by Denarié [72] on wedge splitting tests at different stages of the crack propagation.

In the case of an initially homogeneous microstructure, the relative relaxation properties do not depend on the internal porosity (the absolute relaxation is proportional to the porosity). This was expected from Alfrey's theorem [73], which states that for an homogeneous viscoelastic material, the space and time response are uncoupled. In the case of ageing paste, as Poisson's ratio changes, Alfrey's theorem would not be valid; in this instance where the paste is assumed to be mature, it should hold. For any sample geometry, the stress (or strain) at any instant in time in the entire sample can be calculated from the initial stress (or strain) state multiplied by the relaxation (or creep) function of the material. Therefore, the macroscopic relaxation function of a damaged homogeneous material cannot be accelerated because of the presence of the micro-cracks. This result is consistent with other simulations in the literature [74].

We find in the case of the concrete microstructure an acceleration of the apparent relaxation, with a similar trend as in the experiments. This trend appears even with an approximate damage pattern, and the material properties for the cement paste and aggregates might not be representative of the materials used in the experiments. It seems therefore that the (macroscopic) acceleration of the relaxation process in damaged concrete is related to the interaction between the cracks and the aggregates. More specifically, the presence of crack in the paste would increase the deformation in the paste, and therefore increase the amplitude of the relaxation in that phase. From a macroscopic perspective, that would be seen as an acceleration of the stress relaxation. If the damage were located in the aggregates (as it is the case for example in ASR [75]), a different trend might be observed.

An important limitation of this work is it's 2D nature. Although the observations described, notably on the role of the microstructure in the apparent creep relaxation are generally true, it is not clear that the magnitude described in these results can be transposed to 3D. The observations should therefore only be taken as quantitative where the 3D nature of the stress field is not a dominant factor. If the distribution of stress concentrations due to the PSD is the same, and the connectivity of the creeping phase is preserved, as is likely the case in a mortar microstructure, we expect the $2 \mathrm{D}$ and $3 \mathrm{D}$ simulations to match.

\section{Conclusion}

We proposed a simple model for the creep of the cement paste, aimed at usage in mesoscale simulations of long-term behaviour and degradation. The model accounts for irrecoverable creep as well as the effects of temperature and relative humidity. The material parameters are easily read on a creep curve with an unloading step. We showed that the model provides an accurate concrete creep behaviour after upscaling.

Using this model in meso-scale simulation of concrete creep showed the impact of microstructure on macroscopic behaviour. Particle shape and orientation might induce anisotropy in the macroscopic behaviour, and that a larger proportion of smaller particles tends to reduce the macroscopic creep. This second effect is similar to previous reports of meso-scale damage and fracture simulations. It is therefore likely to be critical in the modelling of coupled creep and degradation. This is of particular importance in three-dimensional models, for which computational resources limit the ratio between the smallest and largest aggregates in the simulation, and forces the separation of the particle size distribution across two separate scales, even though such separation doesn't exist in reality.

We highlight the influence of the micro-mechanical interaction between the aggregates and the cement paste in damaged concrete microstructures: micro-cracks in the cement paste tend to accelerate the apparent stress relaxation of concrete. This effect was shown by independent experimental studies in the literature, but it is the first time to the authors knowledge that this effect is explained from a micro-structural perspective, as such acceleration would not be found in purely homogeneous materials.

In future works, the proposed visco-elastic model will be complemented with a rate-dependent damage model, in order to capture the shift in failure mode with the loading rate, as well as the occurrence of delayed failure under load. Further developments could focus on the difference between creep in tension and in compression, or the difference between volumetric and deviatoric creep, provided sufficient information on the particle shape and orientation are given. 

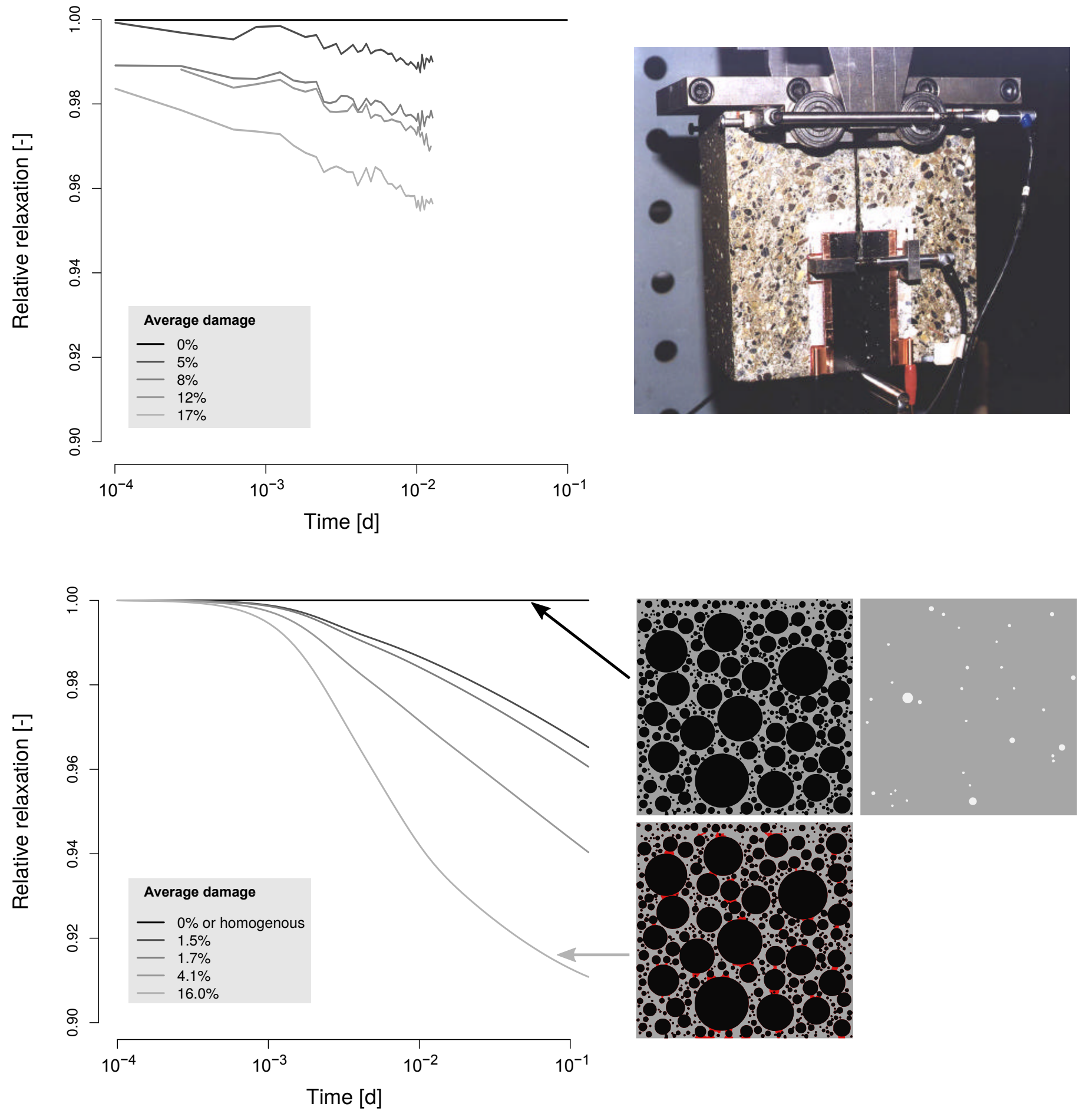

Figure 7: Experimental (top) and numerical (bottom) relaxation curves at different stages of the degradation process. All curves are normalized with respect to the undamaged relaxation curve. Examples of microstructures are provided for different levels of damage or porosity (aggregates in black, cement paste in grey, cracks in red, pores in white). Experimental results and setup from [72]. 


\section{Acknowledgments}

This research is sponsored by the U.S. Department of Energy Light Water Reactor Sustainability Program.

This manuscript has been authored by UT-Battelle, LLC under Contract No. DE-AC05-00OR22725 with the U.S. Department of Energy. The United States Government retains and the publisher, by accepting the article for publication, acknowledges that the United States Government retains a non-exclusive, paidup, irrevocable, world-wide license to publish or reproduce the published form of this manuscript, or allow others to do so, for United States Government purposes. The Department of Energy will provide public access to these results of federally sponsored research in accordance with the DOE Public Access Plan (http: //energy.gov/downloads/doe-public-access-plani?.

\section{References}

\section{References}

[1] E. Schlangen, E. Garboczi, Fracture simulations of concrete using lattice models: computational aspects, Engineering Fracture Mechanics 57 (2) (1997) 319-332.

[2] Z. Wang, A. Kwan, H. Chan, Mesoscopic study of concrete I: generation of random aggregate structure and finite element mesh, Computers \& Structures 70 (5) (1999) 533-544.

[3] E. Denarié, C. Cécot, C. Huet, Characterization of creep and crack growth interactions in the fracture behavior of concrete, Cement and Concrete Research 36 (3) (2006) 571-575.

[4] C. Du, L. Sun, Numerical simulation of aggregate shapes of twodimensional concrete and its application 1, Journal of Aerospace Engineering 20 (3) (2007) 172-178.

[5] F. Grondin, H. Dumontet, A. Ben Hamida, G. Mounajed, H. Boussa, Multiscales modelling for the behaviour of damaged concrete, Cement and Concrete Research 37 (10) (2007) 1453-1462.

[6] F. Dupray, Y. Malecot, L. Daudeville, E. Buzaud, A mesoscopic model for the behaviour of concrete under high confinement, International journal for numerical and analytical methods in geomechanics 33 (11) (2009) $1407-1423$

[7] S.-M. Kim, R. K. Abu Al-Rub, Meso-scale computational modeling of the plastic-damage response of cementitious composites, Cement and Concrete Research 41 (3) (2011) 339-358.

[8] S. Shahbeyk, M. Hosseini, M. Yaghoobi, Mesoscale finite element prediction of concrete failure, Computational Materials Science 50 (7) (2011) 1973-1990.

[9] V. Nguyen, M. Stroeven, L. J. Sluys, Multiscale failure modeling of concrete: micromechanical modeling, discontinuous homogenization and parallel computations, Computer Methods in Applied Mechanics and Engineering 201 (2012) 139-156.

[10] A. Guidoum, Simulation numérique 3D des comportements des bétons en tant que composites granulaires, Ph.D. thesis, Ecole Polytechnique Fédérale de Lausanne, ${ }^{\circ} 1310$ (1994).

[11] A. Tran, J. Yvonnet, Q.-C. He, C. Toulemonde, J. Sanahuja, A four-scale homogenization analysis of creep of a nuclear containment structure, $\mathrm{Nu}$ clear Engineering and Design 265 (2013) 712-726.

[12] F. Lavergne, K. Sab, J. Sanahuja, M. Bornet, C. Toulemonde, Investigation of the effect of aggregates' morphology on concrete creep properties by numerical simulations, Cement and Concrete Research 71 (2015) 14-28.

[13] T. de Larrard, B. Bary, E. Adam, F. Kloss, Influence of aggregate shapes on drying and carbonation phenomena in $3 \mathrm{~d}$ concrete numerical samples, Computational Materials Science 72 (2013) 1-14.

[14] I. Maruyama, A. Sugie, Numerical study on drying shrinkage of concrete affected by aggregate size, Journal of Advanced Concrete Technology 12 (8) (2014) 279-288.

[15] C. Dunant, K. Scrivener, Micro-mechanical modelling of alkali-silicareaction-induced degradation using the AMIE framework, Cement and Concrete Research 40 (4) (2010) 517-525
[16] A. Giorla, K. Scrivener, C. Dunant, Influence of visco-elasticity on the stress development induced by alkali-silica reaction, Cement and Concrete Research 70 (2015) 1-8.

[17] T. Wu, İ. Temizer, P. Wriggers, Multiscale hydro-thermo-chemomechanical coupling: Application to alkali-silica reaction, Computational Materials Science 84 (2014) 381-395.

[18] F. Grondin, H. Dumontet, A. Ben Hamida, H. Boussa, Micromechanical contributions to the behaviour of cement-based materials: Two-scale modelling of cement paste and concrete in tension at high temperatures, Cement and Concrete Composites 33 (3) (2011) 424-435.

[19] F. Bernard, S. Kamali-Bernard, W. Prince, 3d multi-scale modelling of mechanical behaviour of sound and leached mortar, Cement and Concrete Research 38 (4) (2008) 449-458.

[20] V. Salomoni, C. Majorana, B. Pomaro, G. Xotta, F. Gramegna, Macroscale and mesoscale analysis of concrete as a multiphase material for biological shields against nuclear radiation, International Journal for Numerical and Analytical Methods in Geomechanics 38 (5) (2014) 518-535.

21] A. Giorla, M. Vaitová, Y. Le Pape, P. Štemberk, Meso-scale modeling of irradiated concrete in test reactor, Nuclear Engineering and Design 295 (2015) 59-73.

[22] Z. P. Bažant, R. Gettu, Rate effects and load relaxation in static fracture of concrete, ACI Materials Journal 89 (5).

[23] I. Fischer, B. Pichler, E. Lach, C. Terner, E. Barraud, F. Britz, Compressive strength of cement paste as a function of loading rate: Experiments and engineering mechanics analysis, Cement and Concrete Research 58 (2014) 186-200.

[24] S. Shah, C. Ouyang, S. Marikunte, W. Yang, E. Becq-Giraudon, A method to predict shrinkage cracking of concrete, ACI materials journal 95 (4).

[25] S. Altoubat, D. Lange, Creep, shrinkage, and cracking of restrained concrete at early age, ACI Materials Journal 98 (4).

[26] F. Benboudjema, F. Meftah, J. Torrenti, Interaction between drying, shrinkage, creep and cracking phenomena in concrete, Engineering structures 27 (2) (2005) 239-250.

[27] A. Giorla, Y. Le Pape, C. Dunant, Computing creep-damage interactions in irradiated concrete, Journal of Nanomechanics and Micromechanics (2017) 04017001.

[28] Z. Bažant, A. Hauggaard, S. Baweja, F. Ulm, Microprestress-solidification theory for concrete creep. I: Aging and drying effects, Journal of Engineering Mechanics 123 (11) (1997) 1188-1194.

[29] M. Vandamme, F. Ulm, Nanogranular origin of concrete creep, Proceedings of the National Academy of Sciences 106 (26) (2009) 10552-10557.

[30] P. Rossi, J. Tailhan, F. Le Maou, L. Gaillet, E. Martin, Basic creep behavior of concretes investigation of the physical mechanisms by using acoustic emission, Cement and Concrete Research 42 (1) (2012) 61-73.

[31] I. Pignatelli, A. Kumar, R. Alizadeh, Y. Le Pape, M. Bauchy, G. Sant, A dissolution-precipitation mechanism is at the origin of concrete creep in moist environments, The Journal of Chemical Physics 145 (5) (2016) 054701 .

[32] D. Gawin, F. Pesavento, B. Schrefler, Modelling creep and shrinkage of concrete by means of effective stresses, Materials and Structures 40 (6) (2007) 579-591.

[33] M. Jirásek, P. Havlásek, Microprestress-solidification theory of concrete creep: Reformulation and improvement, Cement and Concrete Research 60 (2014) 51-62.

[34] A. Hilaire, F. Benboudjema, A. Darquennes, Y. Berthaud, G. Nahas, Modeling basic creep in concrete at early-age under compressive and tensile loading, Nuclear Engineering and Design 269 (2014) 222-230.

[35] C. Pichler, R. Lackner, A multiscale creep model as basis for simulation of early-age concrete behavior, Computers and Concrete 5 (4) (2008) 295-328.

[36] S. Scheiner, C. Hellmich, Continuum microviscoelasticity model for aging basic creep of early-age concrete, Journal of Engineering Mechanics 135 (4) (2009) 307-323.

[37] V. Šmilauer, Z. Bažant, Identification of viscoelastic C-S-H behavior in mature cement paste by FFT-based homogenization method, Cement and Concrete Research 40 (2) (2010) 197-207.

[38] M. Vandamme, F. Ulm, Nanoindentation investigation of creep properties of calcium silicate hydrates, Cement and Concrete Research 52 (2013) 38-52.

[39] Q. Do, Modelling properties of cement paste from microstructure: Porosity, mechanical properties, creep and shrinkage, Ph.D. thesis, Ecole Polytech- 
nique Fédérale de Lausanne, $\mathrm{n}^{\circ} 5881$ (2013).

[40] B. Tamtsia, J. Beaudoin, Basic creep of hardened cement paste a reexamination of the role of water, Cement and Concrete Research 30 (9) (2000) 1465-1475.

[41] P. Acker, Swelling, shrinkage and creep: a mechanical approach to cement hydration, Materials and Structures 37 (4) (2004) 237-243.

[42] H. Jennings, Refinements to colloid model of C-S-H in cement: CM-II, Cement and Concrete Research 38 (3) (2008) 275-289.

[43] M. Shahidi, B. Pichler, C. Hellmich, Viscous interfaces as source for material creep: a continuum micromechanics approach, European Journal of Mechanics-A/Solids 45 (2014) 41-58.

[44] A. Bazzoni, Study of early hydration mechanisms of cement by means of electron microscopy, Ph.D. thesis, Ecole Polytechnique Fédérale de Lausanne, ${ }^{\circ} 6296$ (2014).

[45] A. Muller, Characterization of porosity \& C-S-H in cement pastes by ${ }^{1} \mathrm{H}$ NMR, Ph.D. thesis, Ecole Polytechnique Fédérale de Lausanne, n6339 (2014).

[46] M. Irfan-ul Hassan, B. Pichler, R. Reihsner, C. Hellmich, Elastic and creep properties of young cement paste, as determined from hourly repeated minute-long quasi-static tests, Cement and Concrete Research 82 (2016) 36-49.

[47] Z. Bažant, Q. Yu, G. Li, Excessive long-time deflections of prestressed box girders. I: Record-span bridge in palau and other paradigms, Journal of Structural Engineering 138 (6) (2012) 676-686.

[48] C. Cécot, Etude micromécanique par simulation numérique en éléments finis des couplages viscoélasticité-croissance des fissures dans les composites granulaires de type béton, Ph.D. thesis, Ecole Polytechnique Fédérale de Lausanne, n²365 (2001).

[49] A. Neville, Creep of concrete: plain, reinforced, and prestressed, 1971.

[50] A. Giorla, K. Scrivener, C. Dunant, Finite elements in space and time for the analysis of generalised visco-elastic materials, International Journal for Numerical Methods in Engineering 97 (6) (2014) 454-472.

[51] C. Dunant, B. Bary, A. Giorla, C. Péniguel, J. Sanahuja, C. Toulemonde, A.-B. Tran, F. Willot, J. Yvonnet, A critical comparison of several numerical methods for computing effective properties of highly heterogeneous materials, Advances in Engineering Software 58 (2013) 1-12.

[52] R. Le Roy, Déformations instantanées et différées des bétons à hautes performances, Ph.D. thesis, École Nationale des Ponts et Chaussées (1995).

[53] O. Bernard, F. Ulm, E. Lemarchand, A multiscale micromechanicshydration model for the early-age elastic properties of cement-based materials, Cement and Concrete Research 33 (9) (2003) 1293-1309.

[54] O. Zienkiewicz, M. Watson, Some creep effects in stress analysis with particular reference to concrete pressure vessels, Nuclear Engineering and Design 4 (4) (1966) 406-412.

[55] C. Bajer, B. Dyniewicz, Virtual functions of the space-time finite element method in moving mass problems, Computers \& Structures 87 (7) (2009) 444-455.

[56] A. Aili, M. Vandamme, J.-M. Torrenti, B. Masson, Theoretical and practical differences between creep and relaxation poisson's ratios in linear viscoelasticity, Mechanics of Time-Dependent Materials 19 (4) (2015) 537-555.

[57] A. Aili, M. Vandamme, J.-M. Torrenti, B. Masson, J. Sanahuja, Time evolutions of non-aging viscoelastic poisson's ratio of concrete and implications for creep of csh, Cement and Concrete Research 90 (2016) 144-161.

[58] Z. P. Bazant, G. Cusatis, L. Cedolin, Temperature effect on concrete creep modeled by microprestress-solidification theory, Journal of Engineering Mechanics 130 (6) (2004) 691-699.

[59] A. Bengougam, Déformations différées et effets d'échelle des bétons de barrage, Ph.D. thesis, Ecole Polytechnique Fédérale de Lausanne, n²624 (2003).

[60] F. Wittmann, Einfluss des feuchtigkeitsgehaltes auf das kriechen des zementsteines, Rheologica Acta 9 (2) (1970) 282-287.

[61] M. Königsberger, M. Irfan-ul Hassan, B. Pichler, C. Hellmich, Downscaling based identification of nonaging power-law creep of cement hydrates, Journal of Engineering Mechanics 142 (12) (2016) 04016106.

[62] M. Königsberger, B. Pichler, C. Hellmich, Micromechanics of itzaggregate interaction in concrete part $\mathrm{i}$ : stress concentration, Journal of the American Ceramic Society 97 (2) (2014) 535-542.

[63] M. Königsberger, B. Pichler, C. Hellmich, Micromechanics of itz-aggregate interaction in concrete part ii: Strength upscaling, Journal of the American Ceramic Society 97 (2) (2014) 543-551.
[64] C. Dunant, K. Scrivener, Effects of aggregate size on alkali-silica-reaction induced expansion, Cement and Concrete Research 42 (6) (2012) 745-751.

[65] Q. Zhang, R. Le Roy, M. Vandamme, B. Zuber, Long-term creep properties of cementitious materials: Comparing microindentation testing with macroscopic uniaxial compressive testing, Cement and Concrete Research 58 (2014) 89-98.

[66] J. Bolomey, Granulation et prévision de la résistance probable des bétons, Travaux 19 (30) (1935) 228-232.

[67] J. Escoda, F. Willot, D. Jeulin, J. Sanahuja, C. Toulemonde, Estimation of local stresses and elastic properties of a mortar sample by FFT computation of fields on a 3D image, Cement and Concrete Research 41 (5) (2011) $542-556$.

[68] O. Bernard, F. Ulm, J. T. Germaine, Volume and deviator creep of calciumleached cement-based materials, Cement and Concrete Research 33 (8) (2003) 1127-1136.

[69] Z. Grasley, D. Lange, The viscoelastic response of cement paste to threedimensional loading, Mechanics of Time-Dependent Materials 11 (1) (2007) 27-46.

[70] T. Nguyen, S. Nguyen, M. Vu, M. Vu, Effective viscoelastic properties of micro-cracked heterogeneous materials, International Journal of Damage Mechanics 25 (4) (2016) 557-573.

[71] G. Pijaudier-Cabot, Z. Bažant, Nonlocal damage theory, Journal of Engineering Mechanics 113 (10) (1987) 1512-1533.

[72] E. Denarié, Etude expérimentale des couplages viscoélasticité-croissance des fissures dans les bétons de ciment, Ph.D. thesis, Ecole Polytechnique Fédérale de Lausanne, $\mathrm{n}^{\circ} 2195$ (2000).

[73] H. Tsien, A generalization of alfrey's theorem for visco-elastic media, Quarterly of Applied Mathematics 8 (1) (1950) 104-106.

[74] Y.-P. Cao, X.-Y. Ji, X.-Q. Feng, Geometry independence of the normalized relaxation functions of viscoelastic materials in indentation, Philosophical Magazine 90 (12) (2010) 1639-1655.

[75] M. Ben Haha, E. Gallucci, A. Guidoum, K. L. Scrivener, Relation of expansion due to alkali silica reaction to the degree of reaction measured by SEM image analysis, Cement and Concrete Research 37 (8) (2007) 1206-1214. 